\title{
Fragments of Delboeuf and Ebbinghaus illusions: Contour/context explorations of misjudged circle size
}

\author{
DANIEL J. WEINTRAUB \\ University of Michigan, Ann Arbor, Michigan \\ and \\ MICHAEL K. SCHNECK \\ New York University Medical Center, New York, New York
}

\begin{abstract}
Variants of the Delboeuf (concentric circle) display and the Ebbinghaus display (four large nonconcentric inducing circles) were produced by systematically eliminating fractions of the circumferences of the outer inducing circles. For the Ebbinghaus display, various amounts of the inner arcs and/or outer arcs were preserved; a series of dots was sometimes substituted for missing contours. Results are described by the following statements: Contours attract. Larger nonconcentric circles induce size contrast. Rotating an Ebbinghaus variant by $45^{\circ}$ alters size misjudgments. The larger the horizontal (or vertical) extent of the inducing configuration, the smaller the judged size of the test (center) circle. A figural aftereffect of the inducing configuration acts to reduce the perceptual size of the comparison circle when eye movements are permitted between test and comparison circles.
\end{abstract}

The term illusion, because of its connotations of magic and deception, neither of which is germane to the topic at hand, shall be restricted to the title. Informativeness requires its inclusion in the title. At issue are explanations of the Delboeuf anomaly, size misjudgments of a circle in the presence of a concentric circle, and the related Ebbinghaus (Titchener circles) anomaly, which depends upon the presence of nearby nonconcentric circles. The underlying problem is to understand the processes underlying misperceived circle size. The present investigation relies upon displays that are sometimes only distant cousins to Delboeuf and Ebbinghaus figures.

The research was designed to explore elements of a theory proposed on the basis of relatively modest evidence (Cooper \& Weintraub, 1970; Weintraub \& Cooper, 1972) but more recently buttressed by extensive evidence (Weintraub, 1979). In barest outline, the contour/circle-context model asserts that contours attract and that context supplied by the larger or smaller size of nearby nonconcentric circles leads to size contrast. Contour attraction, then, predicts that exterior contours produce overestimation of

Experiment 2 was based on research by the second author for his senior honors thesis in the Psychology Department, University of Michigan. This investigation was supported in part by a United States Public Health Service Research Scientist Development Award (K3-MH-35,253) to the first author, and in part by Grant MH23767 from the National Institute of Mental Health.

Requests for reprints should be sent to Daniel J. Weintraub, Human Performance Center, Perry Building, 330 Packard Road, Ann Arbor, MI 48104 . a circle's size, whereas the contextual effect of larger circles produces underestimation. Thus, the Ebbinghaus anomaly, depicted on the right in Figure 2, is assumed to be influenced by both contour and circle context. The experimental manipulation used to disentangle contour from context in Weintraub (1979) was the inclusion of an arcs display, the $25 \%$ of the circumference of the $\mathrm{Eb}-$ binghaus contextual circles closest to the central circle whose size is to be judged (illustrated in the bottom panel of Figure 2). The arcs display is presumed to lack the contextual component associated with the complete circles.

With the postulation of an additive model of effects, subtracting the size misjudgments of the arcs display from size misjudgments of the equivalent Ebbinghaus display provides an estimate of the contextual effect alone. (See Weintraub, 1979, for details.) The data of Weintraub (1979) were very supportive of the model permitting the integration of seemingly contradictory data already in the literature (Girgus, Coren, \& Agdern, 1972, vs. Massaro \& Anderson, 1971).

The work to be reported owes its inspiration to the $\mathrm{Eb}$ binghaus variants investigated by Morinaga and summarized by Oyama (1960) in his review of Japanese research on geometrical-visual anomalies. We tried to push the Morinaga approach to the limit by systematically removing interesting fragments from Delboeuf and Ebbinghaus displays. A complementary strategy was to try to reconstitute the contextual information by substituting a series of spaced dots for missing contours. Thus, an Ebbinghaus nonconcentric circle can be limned by a set of dots ( 8 or 12 or 16 ) rather than by a continuous line forming the 
circumference. The logic of the experiments is straightforward: Removing contours disrupts contour and context processing. Replacing contours with dots preserves contextual-circle information at the expense of contour information. Therefore, the influences of contour and context can be teased apart by means of comparisons among suitable displays.

A substantial amount of parametric data will be presented. To give away part of the story in advance, the contour/circle-context model, even if it is pointed in the proper direction, is far too rudimentary to explain all of the important regularities that appear in the evidence. Moreover, alternative theoretical notions might do as well or better in explaining aspects of the data. Restle and Merryman (1969) have a version of adaptation-level theory that considers distances between elements of a display as well as the size of contextual elements. Pressey (e.g., Pressey \& Murray, 1976) has proposed assimilation theory, which incorporates a construct called the attentive field. The size of contextual elements and the proximity of contextual elements to the element whose size is to be judged are crucial variables. Uhlarik and Brigell (e.g. , Brigell, Uhlarik, \& Goldhorn, 1977) also consider distance an important variable. They have proposed framing ratio as an explanatory principle-the ratio of the total linear extent of a figure to the extent of the element being judged, which would be, for example, the widest dimension across an Ebbinghaus display divided by the diameter of the central circle. (In the experiments to be described, the linear extent of the frame is equivalent to the framing ratio, since the size of the central circle is fixed.) The framing ratio is a simple measure that turns out to have explanatory power. Unfortunately, the framing ratio tends to grow as contextual circles grow. The concluding theoretical stance may not prove attractive, but you are likely to be interested in the data on which the stance is based. We do hope to convince you that a pet all-purpose process (or even a pet pair) can accommodate only a portion of the data.

\section{GENERAL METHOD}

The test circle is the center circle of a display whose size is misjudged as a consequence of the inducing configuration. There are various categories of inducing configuration. The Delboeuf display consists of an outer concentric circle; for clarity, the redundant term, concentric Delboeuf, will be used. A majority of the displays are variants of the nonconcentric inducing circles of the Ebbinghaus display, fragments consisting of the inner arcs of the inducing circles (those closest to the test circle), outer arcs (those farthest from the test circle), dots to indicate a missing contour, inner arcs plus dots, and so forth. Terms like "arcs" and "dots" imply nonconcentric (Ebbinghaus) fragments unless it is specifically stated otherwise. The size of the test circle is compared with that of the nearby comparison circle. The psychophysical technique was the group version of the staircase (up and down) method. With this procedure, subjects are shown a given stimulus display only once and are asked to judge whether the comparison circle is too large or too small to match the test circle. The comparison circle presented for judging depends upon the previous subject's response to the dis- play in question. If the previous subject judged the comparison circle as being too large, then the diameter is reduced by one step (.5 mm). If the previous subject responded with "too small," then the diameter of the comparison circle is increased by one step. A separate staircase series is maintained for each display, and the subject-bysubject judgments drive the stimulus level within the region of uncertainty (i.e., the region in which subjects are uncertain about whether the comparison stimulus appears too big or too small), permitting the estimation of the point of subjective equality, the point at which the probability of each response equals .5 .

Throughout the series of experiments, the details of experimenting remain relatively fixed. Therefore, a standard method will be described, and deviations will be noted when an experiment is introduced.

\section{Subjects}

The 60 subjects were students at the University of Michigan.

\section{Stimuli}

A set of stimulus cards was prepared with a 20.0-mm-diam test circle centered on each $5 \times 5$ in. $(12.7 \times 12.7 \mathrm{~cm})$ white $(82 \%$ reflectance) card. Contours were drawn in black ( $3 \%$ reflectance) India ink with a line thickness of $0.4 \mathrm{~mm}$. Dimensions, measured from center to center of drawn lines, were verified under $25 \times$ magnification; test circles (and the comparison circles to be described next) were drawn and checked with extra care. Each card contained a particular Delboeuf or Ebbinghaus variant as the inducing configuration circumscribed about the test circle. A set of comparison circles, one to each $5 \times 5$ in. $(12.7 \times 12.7 \mathrm{~cm})$ card, ranging in diameter from 17.0 through $24.0 \mathrm{~mm}$ in .5- $\mathrm{mm}$ steps, was also drawn. When a particular comparison circle was set adjacent to the display to be judged, the center-to-center horizontal distance between comparison and test circles was $5 \mathrm{in} .(12.7 \mathrm{~cm})$. Illuminance on the cards was $90 \mathrm{~lx}$.

\section{Procedure}

One stimulus card and an adjacent card containing a comparison circle were presented in a white card holder at a distance of $1.2 \mathrm{~m}$ from the subject, who viewed the cards binocularly. Fixation was not required. As described previously, the group version of the staircase psychophysical method was used. In accordance with the method, each card containing an inducing figure with a centered test circle was paired with a comparison circle whose size was determined by the previous subject's response to that inducing-test display. The subject was asked to state whether the comparison circle was larger or smaller than the test circle (objective instructions). Each subject provided one judgment for each of the displays presented in a random order determined anew for each subject. The experimenter, behind the card holder, switched cards between trials and recorded the responses.

\section{Data Analysis}

Although a "within-subjects" design is used, with each subject providing a judgment for each display, data to compute a judged size for any subject are lacking. For a given stimulus display, the series of 60 observations, 1 per subject, was partitioned into 10 blocks of 6 observations each. For each block of 6 observations, a Dixon and Massey (1957) staircase mean was computed. Each block can be considered as a composite subject, and the mean for that block, $m$, is the point of subjective equality, the estimate of the comparison circle judged to be equal to the standard circle for that composite subject.

For statistical purposes, the data for an experiment represent a within-subjects design with 10 composite subjects, each tested once with every stimulus display. The $10 \mathrm{~m}$ values are treated as the raw data. The mean of the $10 \mathrm{~ms}$ is the mean judged size plotted in the 
figures, and the standard deviation of the $10 \mathrm{~ms}$ divided by $\sqrt{10}$ is the estimate of \pm 1 standard error of the mean depicted in the figures as a vertical bar about the mean.

\section{EXPERIMENT 1}

\section{Method}

The experiment consisted of 53 stimulus displays. In addition to the control condition, a $20.0-\mathrm{mm}$ naked test circle, there were four basic types of inducing configuration. The first contained two opposing arcs from the concentric Delboeuf inducing circle (illustrated in Figure 1, top panel). The diameter of the concentric arcs was $30 \mathrm{~mm}$ (i.e., the radius of curvature was $15 \mathrm{~mm}$ ). Also included was a double concentric display consisting of two complete outer circles, 30 and $45 \mathrm{~mm}$ in diameter. A second type of display was an inside-out version of the two-arc concentric Delboeuf just described, in which the two arcs were flipped outward so that they no longer enveloped the test circle (illustrated in Figure 1, bottom panel). The radius of curvature of the arcs remained at $15 \mathrm{~mm}$; the closest point of approach between a flipped arc and the test circle was $5 \mathrm{~mm}$. The third type of inducing configuration was the fourlarge-circle version of the Ebbinghaus display, preserving one fragment (either the innermost or the outermost arc of each inducing circle), as illustrated in Figure 2, bottom panel. The diameter of these nonconcentric inducing circles was $40 \mathrm{~mm}$ (radius of curvature $20 \mathrm{~mm}$ ) with proximity at the closest point fixed at $5 \mathrm{~mm}$. The fourth type of display was an Ebbinghaus variant, double arcs; a pair of opposing arcs from each of the four nonconcentric inducing circles was drawn as illustrated in the top panel of Figure 2.

Various orientations of the displays were drawn. Progressing from a zero amount of surrounding contour, the control circle, the arcs were lengthened in $25 \%$ increments toward completed circumferences.

\section{Results and Discussion}

The data from concentric Delboeuf fragments are displayed in the top panel of Figure 1. Contrary to expectations, the particular orientation of the concentric arcs showed no consistent effect. However, as the outer inducing circle progressively surrounded the test circle, judged size increased. (Note that the extra outer concentric circle of the double-concentric Delboeuf added a dollop of judged size.) The outcome is consistent with a contour-attraction hypothesis, and matches the evidence of Weintraub, Wilson, Greene, and Palmquist (1969, Experiment 1 ), who used eight equally spaced small inducing fragments and found that the more complete the contour, the greater the amount of error in judging size. In terms of alternative explanations, completing the concentric inducing circle does not increase the extent of the frame surrounding the test circle, but one might wish to argue that the greater the circumference, the more subjectively circular the inducing figure appears.

The bottom panel of Figure 1 presents the outcome of turning the concentric arcs inside out. As the figure caption explains, the flipped arcs taken together form a given percentage of the standard concentric display. Thus, two completed nonconcentric circles would have been represented along the abscissa at $200 \%$. Again, there is scant evidence that the orientation of the fragments is crucial. However, the amount of circumference makes a difference. The first $25 \%$ produces an increase in judged size

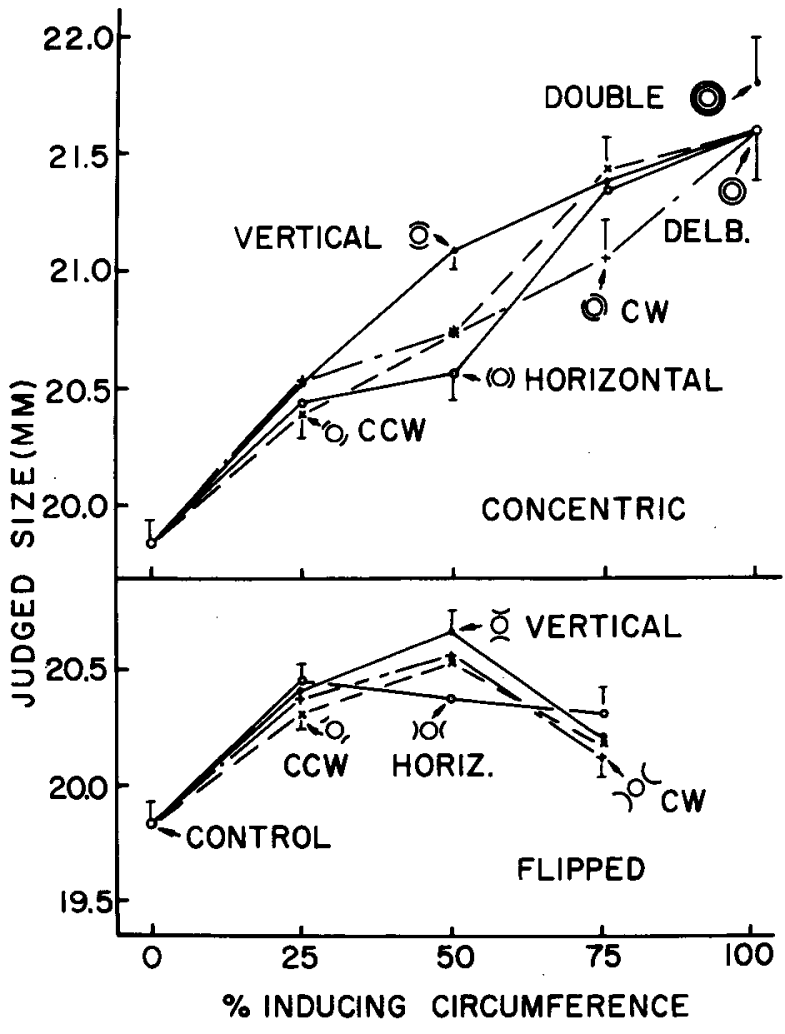

Figure 1. Judged size of the test circle as a function of the orientation of the arcs and the percentage of inducing-circle circumference represented. Top panel: Pairs of opposite arcs from the concentric Delboeuf display. Bottom panel: The concentric arcs flipped inside out (percentage of circumference measured before flipping).

over the control condition that is equivalent to the increase found with concentric arcs. From there, the functions in the lower and upper panels diverge. There is no doubt that if data had been gathered to $200 \%$ circumference, an Ebbinghaus display with two outer circles, the function would have continued to decrease below the judged size of the control condition. (See Massaro \& Anderson, 1971, Figure 2, for a plot of confirming evidence.) It can be concluded that the circle-context effect is entering to counter the initial contour attraction as Weintraub (1979) argued. A critic would note that the extent of the frame is increasing also.

A glance at the data gathered from Ebbinghaus nonconcentric arcs shown in Figure 2 will disclose the strong influence of the orientation of the display, which is revealed most strikingly when there is one arc displayed from each of the four inducing circles (bottom panel). The outcome was expected, and it confirms Weintraub and Cooper (1972, p. 195), who were skeptical enough of their original discovery to check it again before publishing. The effect is curious because it appears to influence whatever type of error is salient. The control condition ( $0 \%$ circumference) specifies zero influence of the inducing figure upon size misjudgments, and rotating the contours of any inducing figure $45^{\circ}$ so that they no longer intersect the horizontal and vertical axes of the display 


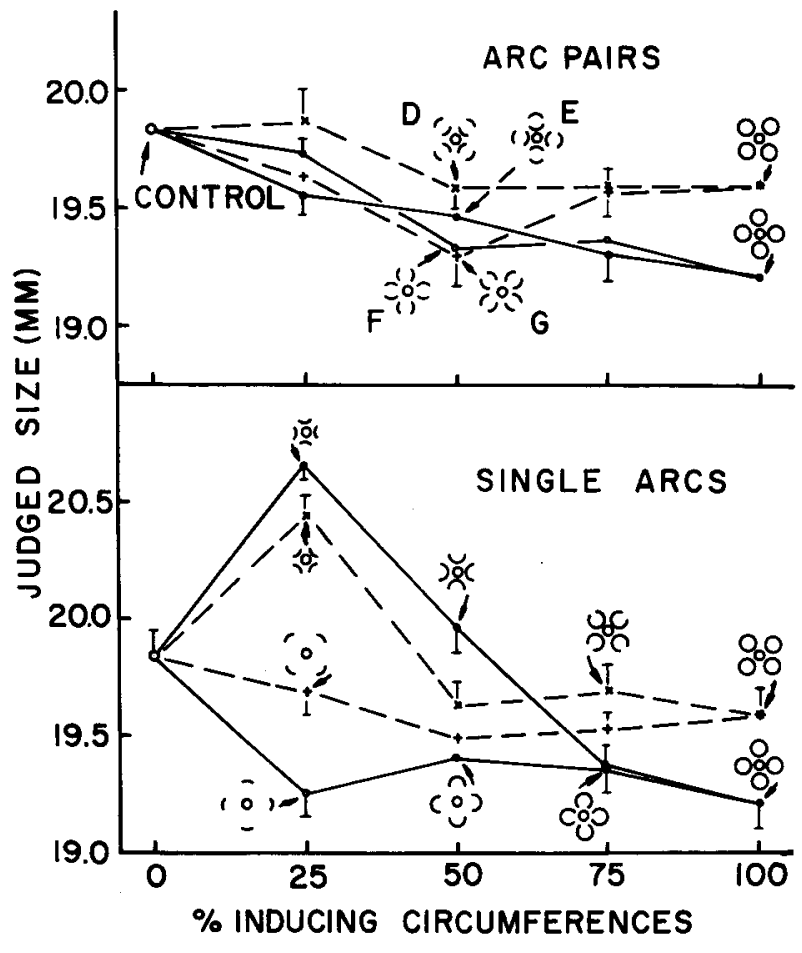

Figure 2. Judged size of the test circle as a function of the orientation of the display and the percentage of each inducing circumference represented. Top panel: Pairs of opposite arcs from each nonconcentric circle constituted the Ebbinghaus variants. Bottom panel: One arc, either inner or outer, from each nonconcentric circle constituted the Ebbinghaus variants.

moves judgmental errors toward those of the control. Size overestimations drop; size underestimations rise. Compare pairs of displays with the same percent circumference in the bottom panel of Figure 2; these are identical except for orientation. The outcome is an interesting variation of the meridional effect (Appelle, 1972; Sekuler, 1974). Judgments are closer to veridical when the horizontal and vertical visual axes are uncluttered by contours. The same result obtains in the top panel of Figure 2, but it is less striking since there is no size overestimation. In addition, for two of these displays (labeled $F$ and $G$ ), neither orientation has inducing contours that intrude across the horizontal and vertical axes.

There is grist for many theoretical mills in Figure 2. In the bottom panel, for inner inducing arcs, the contour/circle-context hypothesis predicts the outcome. Contour attraction is gradually overridden by contrast induced by the larger contextual circles; the filling in of these circles increases subjective circularity. For outer inducing arcs, however, the data point toward contour repulsion. The outer arcs are not concentric; considered as a gestalt, they form a rounded square with its sides missing. At its widest, the rounded square has a width ratio of $51 / 2$ to 1 with respect to the test circle. Data on the concentric Delboeuf display have shown that overestimation gives way to size underestimation at diameter ratios of 5 to 1 or so (Morinaga, 1935, cited by Oyama, 1960). Note that the size underestimation takes place at $25 \%$ circumfer- ence with relatively little change thereafter as the circumferences are completed. One may argue that as the outer arcs are completed, increasing contour attraction is balanced by the induced contrast from increasingly subjective circularity.

In the top panel of Figure 2, a framing explanation would claim that all frames are nearly equally large, so that underestimation of all displays would be about the same. A contour/circle-context explanation would claim that the judged sizes produced by inner-contour attraction should be larger for displays $D$ and $E$ than for $F$ and $\mathrm{G}$ that lack inner contours. The prediction does not hold so well, however, for $25 \%$ circumferences.

\section{EXPERIMENT 2}

A new sample of 60 subjects was used; the eye-todisplay distance was changed to $1.5 \mathrm{~m}$.

\section{Method}

Stimuli. A set of 76 displays was prepared, most of which can be categorized into five groups, corresponding to Figures 3, 4, 5, 6 top, and 6 bottom. Several of the displays from Experiment 1 were redrawn to be presented again. The mean size judgments for the old data and new data, which included a control display, were so close that old and new data were treated as equivalent. Therefore, in Figures 3 through 6, whenever it was useful to do so, means from Experiment 1 were included (each such plotted mean appears in parentheses) and used to determine the shape of functions.

With a few exceptions, the displays consisted of fragments of the four-large-circle Ebbinghaus display, 40-mm-diam inducing circles surrounding a 20 -mm-diam test circle, with a 5-mm gap between the closest point of the complete inducing circle and the test circle, as illustrated in Figure 3 (the same Ebbinghaus configuration used in Experiment 1). The largest dimension of a complete Ebbinghaus display was $110 \mathrm{~mm}$. For some displays, those whose data are presented in Figures 4 and 5, small black dots (with unfilled centers) were placed at selected points where contours of inducing circles were missing. The dots were $1.5 \mathrm{~mm}$ in diameter (dry-transfer dots put in place by rubbing; Roll Deca-Dry, Style 4RDC1-black).

For the displays of Figure 4, an inner arc of $25 \%$ circumference from each inducing circle was represented. The 3-dot display had 1 dot at the midpoint of each missing $25 \%$ of arc. (Taken together, these 12 dots from all four circles form their own gestalt, a surrounding square, as shown in Figure 4.) The 5- and 11-dot displays substituted equally spaced dots for the missing $75 \%$ of arc in each inducing circle.

For the displays of Figure 5, each inducing circle is represented entirely by dots $2,3,4,8,12$, or 16 . The displays with 2,3 , and 4 dots per inducing circle are illustrated in Figure 5; these displays do not give the impression of four inducing circles. The use of 8 , 12 , or 16 dots (which are equally spaced) does provide the impression of four inducing circles.

Inducing configurations or portions thereof, shown in Figure 6, were drawn not in black, but with Grumbacher Gamma Neutral Gray paints (M. Grumbacher, Inc.) thinned with water and used like ink in a ruling compass. Light gray lines were drawn with Gamma 1 paint, $57 \%$ reflectance, and dark gray lines with Gamma 5 paint, $12 \%$ reflectance.

Procedure. After completing the staircase judgments, each subject was asked to rate the subjective circularity of a selected subset of displays by responding to the following instructions: "Please indicate on the basis of one to six the degree of circularity that the inducing figure has. The inducing figure is defined as everything 
on the card except for the inner test circle. One is most circular and six is least circular." In retrospect, it is clear that these ratings have built-in ambiguities. One cannot be sure how the subject is grouping fragments. For example, the four $25 \%$ outer arcs, as depicted in the top of Figure 6, might be grouped into a reasonably circular large configuration. Or each arc might itself be considered not very circular. For this particular display only, subjects were asked, "Do you see it as parts of four circles or parts of one larger circle?" (Parts of four circles was the response of 45 of the 60 subjects.)

\section{Results}

Given the authors' investment in a contour/circlecontext hypothesis, the metamorphosis of inner arcs into full-fledged Ebbinghaus inducing circles is of special interest. The course of assumed contour attraction received close scrutiny via increasing arc length, in what was estimated to be the critical region, by increments of $1 / 32$ of the circumference of the inducing circles. In addition, the surprising orientation effect was put to another test. Figure 3 shows the outcome with means from Experiment 1 supplementing the new data. The data are completely consistent with previous findings. Arc displays produce size overestimation that gives way to size underestimation. The orientation effect is strong. Again, the contour/circle-context notions explain well. With these more extensive data, it can be observed that size overestimation begins to decline when the arcs have traced only a fraction of the circumferences of the four inducing circles. Subjects' circularity ratings corroborate one's own intuitions. On the scale of 1 to 6 , with 6 as least circular, circularity ratings for circumference proportions of $4 / 32$, $8 / 32,12 / 32$, and $16 / 32$, with arcs along horizontals and verticals, were 5.00, 4.33, 3.77, and 3.17, respectively. Frame sizes, it should be noted, progressively increase with an increase in the amount of circumference represented.

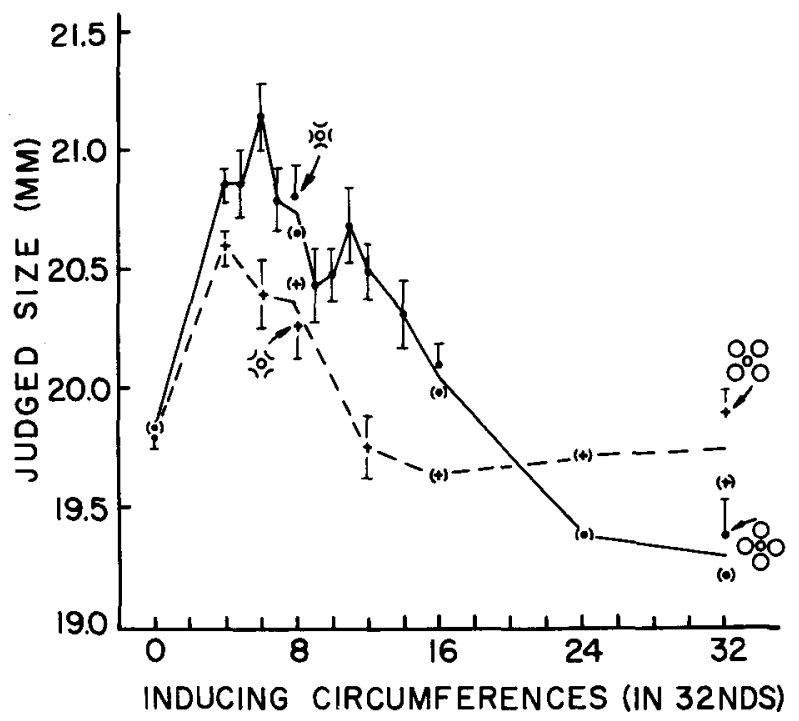

Figure 3. Judged size of the test circle as a function of the percentage of each inducing-circle circumference represented (inner arc) and orientation of the display.

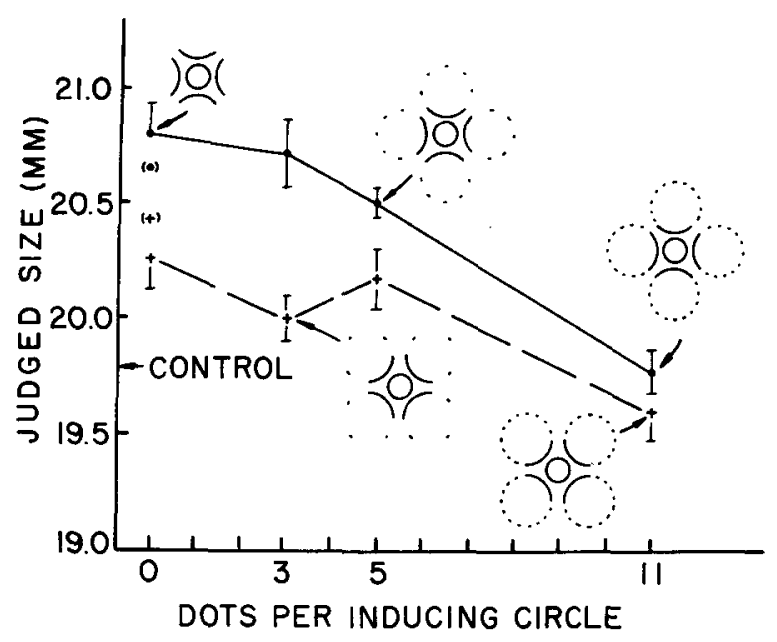

Figure 4. Judged size of the test circle as a function of the orientation of the display and the number of dots used to represent the missing $75 \%$ of each nonconcentric-circle circumference.

Figure 4 shows the effects of adding increasing numbers of dots to the inner arcs. As the number of dots per inducing circle increased, size overestimation decreased toward the size judgments for the control stimulus. The orientation effect for size overestimation is again evident. It is likely that with even greater dot densities the usual contrast plus orientation reversal effects would have been found. A circle-size contrast explanation for these data is a reasonable one. As the number of dots per inducing circle increased, subjects rated the displays as increasingly circular; 3,5 , and 11 dots (circles oriented horizontally and vertically) were rated $4.63,2.97$, and 2.60 , respectively; 3,5 , and 11 dots (rotated orientation) were rated 4.93, 2.97, and 2.72, respectively. The Ebbinghaus display with complete circles horizontally and vertically was rated at 2.01; the concentric Delboeuf was rated at 1.05. Frame extent, as defined by the farthest-out dots, does not increase as the number of dots increases.

The data in which only dots form the inducing configuration are plotted in Figure 5. Without the inner arcs, there is little size overestimation, which is consistent with a contour-attraction hypothesis with missing contours. The two major characteristics of these data are the orientation effect and the minor amount of size misjudgment. Subjects rated the circularity of displays as might be expected. For 2, 3, and 4 dots per inducing circle, circularity ratings were $5.45,3.97$, and 5.32 for the inducing circles oriented horizontally and vertically, not very circular (see the illustrations in Figure 5). For 8, 12, and 16 dots, the same orientation, the ratings were $2.58,2.27$, and 2.37; that for the complete Ebbinghaus display was 2.01 . A contour/circle-context theory predicts that in the absence of the inner-arc contour attraction to offset size contrast, there should be size underestimation, especially for 12 and 16 dots, which give four well articulated dotted inducing circles. An explanation for the mildness of the effect based upon similarity can be invoked. Size contrast is most powerful among similar elements. The inducing 
circles are composed of open dots, the test circle a continuous line contour, so that one expects less size contrast. (The same explanation can be applied to the arcplus-dots inducing circles for the data of Figure 4.) From 4 dots per inducing circle onward, frame extent changes very little and a framing theorist would argue that judged size should also change very little. Such is the case.

There is abundant evidence for the concentric Delboeuf display that the less the reflectance contrast of an inducing circle, the less the contour-attraction effect (Cooper \& Weintraub, 1970; Weintraub et al., 1969). Thus, fading out a contour will gradually eliminate size misjudgments. The rationale for presenting the displays whose data appear in Figure 6, bottom panel, is that if contour attraction is a factor in the Ebbinghaus display, then the inner arcs, labeled I, are critical, and their fading should give greater size underestimation than equivalent fading of either the outer $(\mathrm{O})$ arcs or side $(\mathrm{S})$ arcs. The variable plotted along the abscissa gives the figure-ground contrast of the dark ink on white cards. The reflectance of the ink in question was divided by the reflectance of the background. This ratio was subtracted from 1 to give reflectance contrast. There are no differential effects at all; the fading out of any portion of the circumference has, for practical purposes, no effect on size misjudgments.

The data in the top panel of Figure 6 are difficult to interpret. Increasing the reflectance contrast of the inducing configuration was intended to increase contour attraction. Contour attraction must increase judged size. Both configurations have frame extents of $110 \mathrm{~mm}$, which might account for the overall size underestimation, but reflectance contrast appears to act in opposite directions; one function rises and the other falls.

\section{EXPERIMENT 3}

A new sample of 64 subjects was solicited. For a given stimulus display, the up-and-down series composed of one

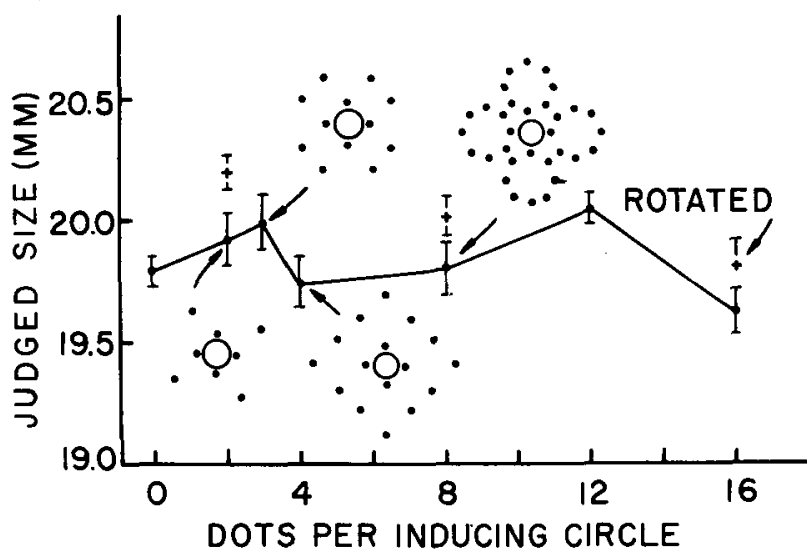

Figure 5. Judged size of the test circle as a function of the orientation of the display and the number of dots used to represent each nonconcentric inducing circle.

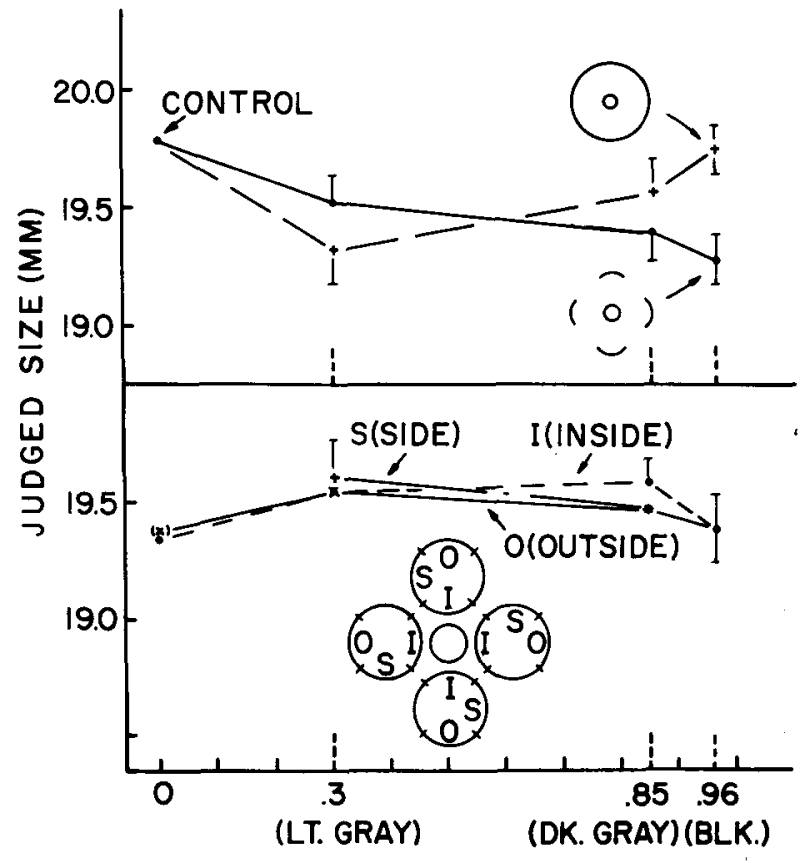

REFLECTANCE CONTRAST

Figure 6. Judged size as a function of reflectance contrast and type of display. Top panel: A nonconcentric-arcs display versus a concentric Delboeuf display with the same cardinal-axes frame extent. Bottom panel: Fading in either inner, outer, or side nonconcentric arcs.

judgment per subject was partitioned into eight blocks of eight observations each for analysis. The datum for a composite subject consisted of the mean judgment for the eight actual subjects comprising that block.

\section{Method}

There were 15 displays, illustrated in Figure 7, which included a control display. As usual, all test circles were $20.0 \mathrm{~mm}$ in diameter. In Displays 3 and 4, the radius of curvature of the outer connecting arcs was $32.5 \mathrm{~mm}$, with a $3.3-\mathrm{mm}$ contour separation between test circle and arcs at their closest points of approach. For the other displays, the closest points of approach between inducing contours and test circles was 5 mm (exception: inner circle, Display $11,31 / 3 \mathrm{~mm}$ ). The outer square in Displays 5 through 8 was $30 \mathrm{~mm}$ on a side. For Displays 7 and 8 , only the middle $20 \mathrm{~mm}$ of a side was drawn. In Displays 9, 10, and 11, the concentric circles were $30 \mathrm{~mm}$ in diameter, with the inner circle of Display 11 being $131 / 3 \mathrm{~mm}$ in diameter and with $50 \%$ of every circumference represented. The squares in Displays 12 through 15 were $25 \mathrm{~mm}$ per side.

\section{Results and Discussion}

Displays with close-in contours, 2 through 11 , led to size overestimation. The concentric Delboeuf with all of its contour at $5-\mathrm{mm}$ proximity produced maximum overestimation as expected, since an inducing-to-test-circle diameter ratio of 3 to 2 was predicted to provide maximum error according to the consensus achieved by previous research. Less than complete concentric contours led to less error; compare Displays 9 and 10 with 2, Display 6 with 8 , and Display 5 with 7 . Also, Displays 3 through 


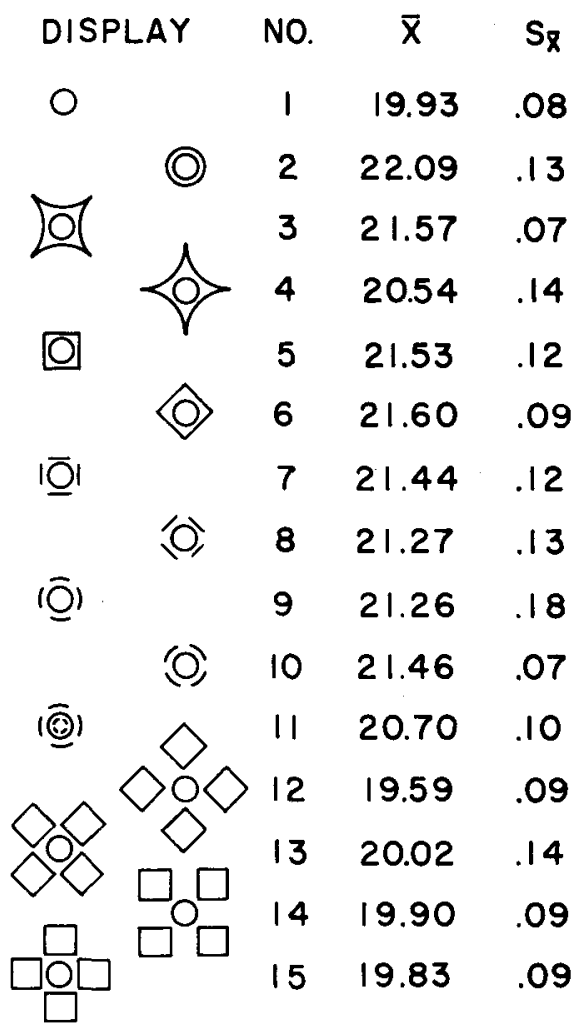

Figure 7. The displays and data of Experiment 3.

8, which have almost all of their inducing contours greater than $5 \mathrm{~mm}$ distant from the test circle, produced less overestimation than the concentric Delboeuf display. There is the usual orientation effect for missing nonconcentric contours ( 7 vs. 8) and for distant portions of contour ( 3 vs. 4), but not for relatively close-in and closed contours ( 5 vs. 6), and a reversal for the four-fragment concentric cases ( 9 vs. 10). In Display 11, it appears that the added inside contour fragments counteract a portion of the original contour effect (compare 9 with 11). These data are consistent with a contour-attraction hypothesis for closein contours.

Displays 12 through 15 , the inducing squares, give evidence of an orientation effect (compare Display 12 with 14 and Display 13 with 15 ). Three of the four do not differ appreciably from judgments of the test circle alone. A size-contrast hypothesis would explain the result by pointing to the modest size difference between inducing squares and test circles, and to the fact that squares and circles are dissimilar in shape and thus less susceptible to size comparisons. A framing hypothesis would claim that the modest frame extents of these displays explains the lack of large errors of over- or underestimation.

There is strong evidence that the inner-arcs $25 \%$ circumference Ebbinghaus display does not provide size overestimation when the display is fixated at a point midway between the centers of the test and comparison circles (Cooper \& Weintraub, 1970). The most salient differ- ence between the displays in the Cooper and Weintraub studies and in the studies reported herein is that the former displays were luminous stimuli in completely dark surrounds rather than black ink on white cards. Another difference is that the horizontal visual angle from the far edge of the comparison circle on one side to the far edge of the inducing contour surrounding the test circles on the other was much reduced for the fixation study in order to move test and comparison circles closer to the central fovea. Under such conditions, size overestimation (reduced in magnitude) was obtained for the concentric Delboeuf display, but there was a reversal to size underestimation for the arcs of the Ebbinghaus display. An important question was whether concentric contours and nonconcentric contours induce fundamentally different effects, and supplementary experiments requiring fixation were accordingly undertaken.

\section{EXPERIMENT 4}

A new sample of 60 subjects was drawn. (Statistically, the data for each display were treated as 3 composite subjects, each based on the judgments of 20 actual subjects.)

\section{Method}

The two displays were the usual concentric Delboeuf display (test circle $20.0 \mathrm{~mm}$ in diameter and inducing circle $30 \mathrm{~mm}$ in diameter) and the $25 \%$ inner-arcs version of the Ebbinghaus display in the rotated orientation (inducing contours oriented obliquely) with the usual 20.0-mm-diam test circle and 20-mm radius-of-curvature arcs, but a 4(rather than 5)-mm contour separation at the closest point of approach between inducing arcs and test circle. Each display and each comparison circle were drawn in black ink on a $3 \times 5$ in. (76.2 $\times 127 \mathrm{~mm}$ ) filing card (82\% reflectance), long side set vertically. Comparison circles were presented on the left, test circles on the right. The comparison and test circles were not centered on their respective cards but offset horizontally toward each other so that the center-to-center distance between a comparison and test circle pair was $76 \mathrm{~mm}$. A grain-of-wheat bulb midway in the center-tocenter distance served as a fixation point. Eye-to-display distance was $1.25 \mathrm{~m}$, so that the horizontal extent of the arcs display plus comparison circle subtended no more than $5^{\circ}$ of visual angle. Stimuli were presented at one end of a dark black box; the subject peered at the grain-of-wheat fixation bulb from a headrest at the other end. The display was illuminated for $1 \frac{1}{2} \mathrm{sec}$; the subject then responded.

\section{Results}

For the concentric Delboeuf display, the mean size judgment was $21.07 \mathrm{~mm}$ (standard deviation of the mean, $.27 \mathrm{~mm}$ ); for the concentric Delboeuf displays from Experiment 1 , the mean size judgment was $21.61 \mathrm{~mm}$ ( $S D$, $.20 \mathrm{~mm}$ ), from Experiment 2, $21.22 \mathrm{~mm}(S D, .12 \mathrm{~mm})$, not previously reported, and from Experiment 3, $22.09 \mathrm{~mm}(S D, .13 \mathrm{~mm})$. The concentric Delboeuf size overestimation is smallest with fixation.

For the arcs of the Ebbinghaus display, the mean size judgment using fixation was $19.35 \mathrm{~mm}(S D, .37 \mathrm{~mm})$; the equivalent display from Experiment 1 was $20.44 \mathrm{~mm}$ $(S D, .08 \mathrm{~mm})$ and from Experiment 2, $20.26 \mathrm{~mm}(S D$, $.13 \mathrm{~mm}$ ). Fixation led to size underestimation, although the results from the free-viewing conditions of the first 
two experiments showed clear size overestimation. The fixation results lie in the opposite direction despite the fact that the arc contours in Experiment 4 are $1 \mathrm{~mm}$ closer to the test circle for which greater contour attraction would be predicted.

These outcomes, with black ink on white cards, corroborate the Cooper and Weintraub (1970) data for luminous stimuli on dark surrounds. Note also the increased variability; the standard deviation of the mean for a fixation condition is larger than for the equivalent free-viewing situation.

\section{EXPERIMENT 5}

Experiment 4 differed in many respects from the preceding three studies. A salient dissimilarity was that the visual field was dark except during the 1.5 -sec viewing of the display. For greater comparability with the initial experiments, an Ebbinghaus-arcs display was selected from Experiment 2 and tested under fixation and freeviewing conditions. A new sample of 60 subjects was used (with the data treated in the standard way, 10 composite subjects, each composed of 6 actual subjects).

\section{Method}

The Ebbinghaus arcs display from Experiment 2 that gave the greatest size overestimation, the $6 / 32$ arcs in the standard (arcs horizontal and vertical) orientation, was selected. The cards were trimmed so that the center-to-center horizontal distance between comparison circle on the left and test circle on the right was $65 \mathrm{~mm}$. Halfway between was a small $(2 \times 2 \mathrm{~mm})$ plus sign for fixation. The cards were presented in a white holder, with the subject's head placed in a headrest to hold eye-to-display distance at $1.80 \mathrm{~m}$. These dimensions kept the total display, comparison, test, and inducing portions, within a horizontal visual angle of $3^{\circ}$. Pollack has argued that this visual angle ought to be no greater than $3^{\circ}$ (Sjostrom \& Pollack, 1972). A vertical slot in a white cardboard mask obscured all but the fixation mark. The experimenter removed the mask manually for $2 \sec ( \pm 1 / 2 \mathrm{sec})$, then replaced it while the subject maintained fixation. The subject then provided a judgment. After the fixation trial, while the subject's eyes were closed, the same display was paired with the appropriate comparison stimulus for a freeviewing judgment. The subject was then permitted an unrestricted view of the display and asked for a second judgment. As usual, separate staircase series were maintained for the fixation and freeviewing judgments. The fixation judgment was always made first on the hypothesis that, especially for naive subjects, it would be difficult to hold fixation after having already been permitted to move one's eyes.

\section{Results}

The mean for free viewing was $21.09 \mathrm{~mm}$ (standard deviation of the mean, .11 mm); it was $21.13 \mathrm{~mm}$ ( $S D$, $.14 \mathrm{~mm}$ ) in Experiment 1. The new and old values are quite close. For the same display under fixation conditions, the mean was 20.93 ( $S D, .15 \mathrm{~mm}$ ), smaller, to be sure, but a difference in means of only $.16 \mathrm{~mm}$. The data represent a within-subjects design with each of the 10 composite subjects providing a judgment (the mean of 6 actual subjects) under each condition. (Although every one of the 60 subjects provided a pair of judgments, in- sufficient information exists to assign a pair of judged sizes to each.) The differences between means for free viewing and fixation for each composite subject provide 10 differences for a $t$ test, and $t=1.67$, which does not quite meet a one-tailed .05 level criterion of $1.83(9 d f)$. The statistical test is legitimate, but conservative, since there were 60 actual subjects. The main point, however, is that although the outcome is in the predicted direction, it is not the whopping difference expected. Fixation did not reverse size overestimation to size underestimation, as it did in Experiment 4. The display was intentionally selected for the largest size overestimation so that obtaining a reversal to underestimation under fixation conditions would necessitate a larger reduction in judged size. Requiring fixation with luminous stimuli in a black box has produced large reductions of nearly $1 \mathrm{~mm}$ (see Cooper \& Weintraub, 1970, Figure 2). Experiment 4, with blackon-white stimuli, also gave a large reversal. Given the orientation and arc length that provided for large size overestimation, a large drop, but not a reversal, was expected. The modest drop weakens our confidence about the effects of eye fixation, but increases our confidence that nonconcentric contours do not differ fundamentally from concentric contours. In both cases proximity is important. However, only a concentric contour can remain in close proximity to the test circle everywhere.

\section{GENERAL DISCUSSION}

With data galore, open season on theory is declared. The initial aim was to garner support for a tidy twoprocess formulation postulating that contours attract and that context in the form of large inducing circles leads to size contrast. The statement is no sooner committed to paper than it begs for modification: Close-in contours seem to attract. But that proviso is only the beginning. Concerning size misjudgments of circles in the presence of various kinds and amounts of contour, what sort of theory is tenable? We are moved to state that in the face of the extensive evidence, no one-process theory is tenable, and the two-process contour/circle-context theory that motivated the research is clearly too simple. Five effects that are salient will be discussed and then an integration that relies on description will be outlined.

\section{Contour Attraction}

The attraction is a function of proximity, near zero when contour elements are very close, increasing to a maximum when inducing contours are $50 \%$ farther out than the radius of the test circle (diameter ratio of 3:2 for concentric circles), then falling rapidly toward zero as contour distance increases. Most of the evidence is derived from previous studies of the concentric Delboeuf display, and the initial rise in attraction is called the distance paradox. The greater the figure-ground contrast of the inducing concentric circle, the lesser the figure-ground contrast of the test circle; the greater the amount of inducing contour present, the lesser the amount of test-circle con- 
tour present; all lead to evidence that can be interpreted as contour attraction (e.g., Weintraub \& Cooper, 1972; Weintraub et al., 1969). The data of Figure 1, top panel, which show large increases in judged size with increasing amounts of concentric inducing circumference, are in strong agreement. There is a question concerning closein nonconcentric contours. Increasing a nonconcentric arc generally decreases the amount of size overestimation after an initial increase (Figure 1, bottom panel, and Figure 3). The explanation to be defended is that lengthening closein nonconcentric contours adds contour elements that are increasingly farther away from the test circle, and therefore adds only very small increments to attraction, while the additional attraction is being overridden by a framing effect. One of the large disappointments for the contourattraction formulation is the fact that Ebbinghaus displays with $75 \%$ of the circumference of each inducing circle displayed gave only modest differences in size judgments when the innermost $25 \%$ of the arcs was omitted, compared to the outermost $25 \%$ (see Figure 2, bottom). For the standard orientation, there was virtually no difference (corroborated by Morinaga, 1956, 1958, cited by Oyama, 1960 ). There should be a large contour-attraction difference between such pairs of displays. The same reasoning can be applied to the double-arc data in the top panel of Figure 2 and to the data in the bottom panel of Figure 6, where the attempt was made to influence "contour attraction" by varying the figure-ground contrast of various quarter-arcs of the inducing circles (without success). However, the failure to obtain the predicted outcome in every case can be the result of effects acting in contrary directions. Weintraub (1979) presented a strong case that contour attraction opposed the contrast stemming from size differences between test and inducing circles.

\section{Size Contrast}

As a result of the presence of large nonconcentric inducing circles, the test circle tends to appear smaller. Many of the data presented can support the hypothesis. The data of Figure 3, for example, can be explained by claiming that contour attraction of inner arcs is gradually overridden by size contrast as the arcs approach complete circles; more nearly complete circles are more potent inducers of size contrast. The explanation for inducing circles that begin as outer arcs (bottom panel of Figure 2) is that, as the outer arcs approach complete circles, the size-contrast effect is balanced by contour attraction of the increasing amounts of close-in arc. Why, then, do not outer arcs at $25 \%$ circumference show contour attraction? One hypothesis is that contour attraction gives way to contour repulsion when the contours are distant. (We prefer an explanation that attributes this size reduction to the extent of the frame, as discussed in the next section.) Further support for circle-size contrast comes from an experiment that added to the inner arcs of circles that were half the size of the test circle, which increased the judged size of the test circle (Weintraub, 1979). (Since extending the inner arcs extends the frame outward, decreased judged size would be predicted by a frame-contrast explanation.) The data of Figure 4 favor a circle-size contrast explanation: As the number of dots in the inducing configuration increases, the four circles become more differentiated and circle-like, while the extent of the frame remains constant. In the present studies, frame extent and circle-size contrast tended to be confounded. This occurred because a framing effect was not seriously considered as an explanation when the research was designed, and because large inducing circles were selected to make the fragmented displays easier to draw.

\section{Orientation Effect}

When contours of the inducing configuration intersect the horizontal and vertical meridians of the display (and, therefore, the horizontal and vertical meridians of the observer's eye), size overestimation is greater, or size underestimation more pronounced, compared to a $45^{\circ}$ rotation of the same display that leaves the horizontal and vertical axes unobstructed. The result could be interpreted as implying that there must be opposed processes, and the meridional effect magnifies their characteristic errors. The orientation effect is pervasive, except for the concentric displays and inside-out concentric displays of Figure 1. The critical feature of the orientation effect may be the framing extent of the inducing contours along the horizontal (or vertical axis). In this regard, concentric Delboeuf displays and inside-out concentric displays change horizontal or vertical framing extent very little with a change in orientation, and there is little or no effect of orientation (Figure 1).

\section{Cardinal-Axes Framing Extent (Framing Ratio)}

As stated in the introduction, the Brigell et al. (1977) approach postulates that the critical aspect of a display is its framing ratio, the overall extent of a display relative to the portion being judged. In our set of experiments the judged portion of the display, the test circle, was fixed at $20 \mathrm{~mm}$. Therefore, specifying the maximum linear extent from one side of an inducing configuration to the other provides equivalent information. Let us define cardinal-axes framing extent as the largest horizontal or vertical dimension across an inducing configuration. (To obtain the cardinal-axes framing ratio, divide by $20 \mathrm{~mm}$.)

In displays rotated $45^{\circ}$ from the standard orientation, not only were the Ebbinghaus inducing circles or their outer arcs located off the horizontal and vertical axes, but the inducing configuration had less horizontal (and vertical) extent and prompted less size underestimation. For small frames, when inner arcs of the Ebbinghaus display were judged in the rotated orientation, the arcs protruded more, both horizontally and vertically, and prompted less size overestimation. In both instances, then, the orientation with the greater cardinal-axes framing extent produced a smaller judged size; therefore, the cardinal-axes framing extent captures features of the data that the largest extent in any direction does not. A scatterplot of the 
cardinal-axes framing extent versus judged size was made for the 104 displays of the first three experiments. Excluded were control trials (test circle alone) and duplicate data (from the same display used in more than one experiment). There are clusters of data at extent $30 \mathrm{~mm}$ (the concentric Delboeuf) and $110 \mathrm{~mm}$ (the Ebbinghaus display in standard orientation with at least the outer edges marked). There are also many intermediate points. The relationship is strongly linear. Judged size decreases as cardinal-axes framing extent increases. The correlation is -.84 (for .01 level significance, $|r|>.26$ with $d f=$ 102). Thus, cardinal-axes framing extent is a variable that accounts for $70 \%$ of the variance in judged size among the displays selected for these experiments. The displays that deviate most from the linearity trend are instructive: At the largest framing extent, $110 \mathrm{~mm}$, the displays whose data appear in Figure 4 are inner Ebbinghaus arcs with either three or five dots describing the outer portions of the inducing circle. These dots mark a large frame according to the definition, but the size-judgment data correspond very closely to cardinal-axes framing extents measured as though the dots were missing. Might subjects be uninfluenced by three or five dots in making judgments? From Figure 5, the 8-dot display (rotated orientation) and the 12-dot display (standard orientation) provided larger than usual size judgments. At the smallest cardinal-axes framing extent, $30 \mathrm{~mm}$, lay the concentric Delboeuf display and its fragments. The concentric inducing circles with the more complete arcs gave size overestimation that exceeded the linearity trend, especially for the complete concentric Delboeuf. Such variation appears to be along a dimension not captured by framing extent. Finally, several maverick displays led to size overestimation that was greater than average. These included the double concentric Delboeuf display of Experiment 1 (a reverse trend, because frame extent was increased by the additional outer concentric circle) and Displays 2 through 10 of Experiment 3 (illustrated in Figure 7). Three of the latter, Displays 2, 9, and 10, are versions of the concentric Delboeuf display, and 3 through 8 apparently act like concentric Delboeuf displays. Displays 3 through 8 have contours whose point-by-point proximity to the test circle is intermediate between a concentric arc and an inner nonconcentric arc, also suggesting that contour attraction is a factor. The horizontal and vertical cusps of Display 4 give a relatively large cardinal-axes framing extent, but the size judgments are larger than predicted for a frame of this extent, as though subjects were not influenced by the far-out cusps. In sum, the framing ratio or its variants cannot fully explain the data.

\section{Figural Aftereffect}

Previous evidence indicates that under fixation conditions in which eye movements are restricted, compared with the free-viewing situation, size judgments decrease regardless of the nature of the inducing configuration (e.g., Cooper \& Weintraub, 1970). The most surprising occurrence is that inner arcs of the Ebbinghaus display elicit a decrease under fixation from size overestimation to size underestimation, a reversal, as occurred in Experiment 4 using the nonstandard orientation. Cooper and Weintraub (1970) proposed that when eye movements are permitted, figural-aftereffect conditions are established in the region surrounding the comparison circle. When one looks from the test circle to the comparison circle, the aftereffect of the inducing figure is superimposed upon the comparison circle; this leads to contrast, the comparison circle appearing smaller. (There is abundant evidence that regardless of the direction of the error for the simultaneous presentation of all stimulus elements, the figuralaftereffects paradigm of inducing figure first, followed by test and comparison circles together, always leads to contrast [Cooper \& Weintraub, 1970; O'Halloran \& Weintraub, 1977]). If the comparison circle appears smaller than it ought, then a larger comparison circle will be chosen for a size match, leading the experimenter to conclude that the test circle appears larger. Although the outcome of Experiment 5 lay in the proper direction, eye fixation did not produce the predicted large judgmental decrement for the inner arcs of the Ebbinghaus display. We are nevertheless willing to claim that nonconcentric arcs do lead to contour attraction, and that "judged size" is boosted by figural aftereffects generated when eye movements are permitted and is counteracted by framing effects and/or orientation effects.

A rudimentary master theory that explains concentric Delboeuf and Ebbinghaus judgmental errors incorporates four processes. (1) Contour attraction exists between adjacent contours as a function of contour separation. If the inducing contours lie outside the test circle, then an increase in judged size occurs; if inside, then a decrease occurs. (2) The circle-size context effect of nonconcentric inducing circles is one of contrast. With inducing circles larger than the test circle, as in the present experiments, a decrease in judged size occurs; with smaller circles, an increase in judged size occurs. When inducing elements are not circles identical to the test circle in all respects except size, the contrast effect also depends upon similarity between the test and inducing elements. For the Delboeuf display the pair of concentric circles (inducing and test) is assumed to form a gestalt, an annulus, eliminating this contrast effect. (3) The cardinal-axes framing ratio (largest horizontal or vertical extent of the inducing configuration relative to the diameter of the test circle) leads to contrast, a decrease in judged size as the ratio increases. This framing formulation incorporates orientation effects (changes in judged size produced by displays in different orientations) by assessing extent along the cardinal axes. (4) When eye movements are permitted, figural-aftereffect contrast alters the perceptual size of the comparison circle to make it smaller. Therefore, a larger physical comparison circle is selected as a match for the test circle, leading to an apparent increase in the judged size of the test circle. These figural aftereffects will also depend upon contour separations, as in the classic figural aftereffects paradigm.

Informed speculation leads to the representation of the four processes as the functions shown in Figure 8. We 


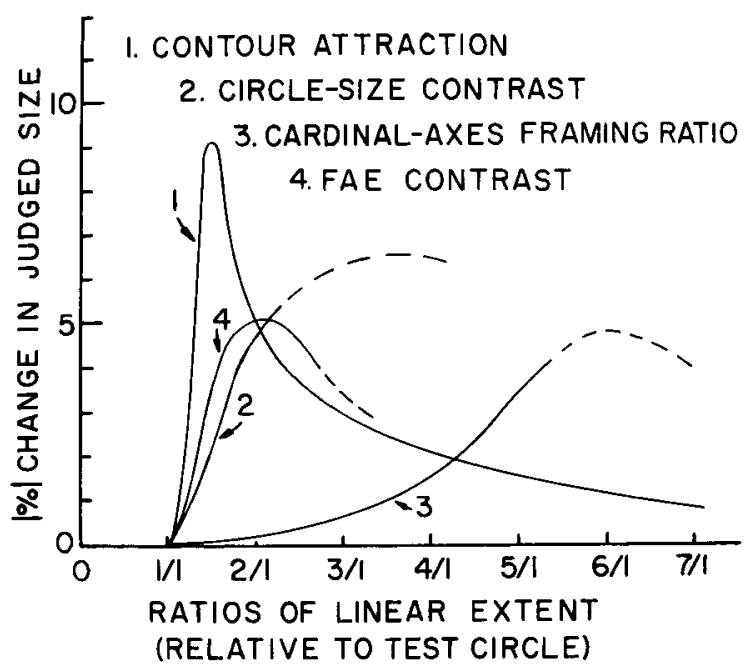

Figure 8. A model of circle-size misjudgments.

claim only to represent trends. The measure along the abscissa is ratios of linear extent. For contour attraction and figural-aftereffect contrast, contour separation can also be represented as a ratio of linear extent, the distance between inducing contours on opposite sides of the center circle divided by center-circle diameter.

The model works wonders with the data of Weintraub (1979). It explains why twice-size inner arcs, when completed into circles of the Ebbinghaus display, lead to a great decrease in size judgments (because the changes in size contrast and framing ratio are acting in the same direction), whereas half-size inner arcs completed into half-size circles give only a modest increase in judged size (because size contrast and cardinal-axes framing ratio oppose one another). It also explains why the arcs of inducing circles that are judged equal in size to the test circle, when they are completed into equal-appearing circles, give a decrease in size judgments (although size contrast is inoperative, the completed circles have a larger framing ratio). Thus, the model can explain regularities in the data of Figure 7 in Weintraub (1979) that were left unexplained. And, as we have tried to show, the set of four processes can encompass much of the new data presented herein.

Our theorizing has consisted of a list of processes (hypothetical constructs), each described by a few lines of print accompanied by a graphic first approximation of each function displayed in Figure 8. (Quantitative predictions would require assigning to each process an algebraic sign that depends upon whether size over- or underestimation of the perceived stimulus should occur, and then summing the effects. The foregoing procedure makes powerful assumptions about additivity, and therefore about lack of interactions among processes, and it depends upon adequate quantification of each function.) In truth, what we have proposed is a rough-hewn description (model), rather than a theory.

However, most theories (higher order explanations) of the Ebbinghaus and Delboeuf anomalies are anemic. The theorist has seized upon one or another salient aspect of the data, promoting this aspect to prime status, and has postulated an explanatory principle. With extensive data at hand, it is easy for us to challenge single-factor positions (or to support one, the framing-ratio position, that had not previously been considered seriously).

A commodious theory by Coren and Girgus (1978; Girgus \& Coren, 1982) addresses geometric visual anomalies and yields useful predictions. Coren and Girgus call their explanation of assimilation-contrast effects a pool and store theory. In brief, information acquired in a single glance (fixation) is pooled (aggregated, averaged) in a short-term sensory register. Pooling acts to average stimulus differences and therefore leads to assimilation; that is, stimulus elements are perceived as more similar (in size, for example) than is warranted by the physical stimuli. Thus, a pair of not very different concentric Delboeuf circles (a diameter ratio of 3 to 2) can be apprehended at a single glance. The inner circle appears larger than it ought; the outer circle appears smaller. Information that is distributed over time because successive fixations are required, or because stimulus elements occur in a temporal sequence, is stored in distinct memory units. Distinguishability among units will be enhanced if stimulus differences from unit to unit are emphasized. As the information is integrated into a percept, the accentuated differences lead to contrast. Thus, when concentric Delboeuf circles differ by a diameter ratio of 5 to 1 , the inner circle appears smaller than it ought, and the outer circle appears larger.

Translating the four-process formulation into pool-andstore terms yields the following: (1) Close-in contours attract, producing assimilation. A single glance is needed, which predicts assimilation. (2) The circle-size context effect is one of contrast. (In the experiments reported, large nonconcentric outer circles reduced the judged size of a smaller center circle.) The display elements cause multiple fixations, which predict contrast. (3) Framing ratio involves the perceptual influence of the widest and tallest extents of a display, producing contrast. The farthest reaches of a display cause multiple fixations, which predict contrast. (4) When eye movements are permitted, figural-aftereffect contrast makes the comparison circle perceptually smaller. Eye movements produce a sequential uptake of information, which predicts contrast.

The theory is meaty. It permits a prediction about the direction (assimilation or contrast) of each of the processes and predicts all four correctly (by chance, 1 out of 16), not a bad theoretical umbrella for a broad range of findings. Note that retinal size, the locations of stimulus elements, and sequencing of stimulus elements are crucial independent variables for pool-and-store theory because they determine whether one fixation suffices for information uptake. The previous statement is meant to demonstrate that the theory is capable of empirical test.

Is the Ebbinghaus-Delboeuf research and theory enterprise worth the effort? Our answer is yes, because we believe that the processes underlying perceptual anomalies 
must surely apply to everyday perception. Literally as well as figuratively, then, it's back to the drawing board.

\section{REFERENCES}

APPELLE, S. (1972). Perception and discrimination as a function of stimulus orientation: The "oblique effect" in man and animals. Psychological Bulletin, 78, 266-278.

Brigell, M., Uhlarik, J., \& Goldhorn, P. (1977). Contextual influences on judgments of linear extent. Joumal of Experimental Psychology: Human Perception \& Performance, 3, 105-118.

Cooper, L. A., \& Weintraub, D. J. (1970). Delboeuf-type circle illusions: Interactions among luminance, temporal characteristics, and inducing-figure variations. Journal of Experimental Psychology, 85, 75-82.

Coren, S., Girgus, J. S. (1978). Seeing is deceiving: The psychol ogy of visual illusions. Hillsdale, NJ: Erlbaum.

Dixon, W. J., \& MASSEY, F. J., JR. (1957). Introduction to statistical analysis (2nd ed.). New York: McGraw-Hill.

Girgus, J. S., \& Coren, S. (1982). Assimilation and contrast illusions: Differences in plasticity. Perception \& Psychophysics, 32, 555-561.

Girgus, J. S., Coren, S., \& AGDERN, M. (1972). The interrelationship between the Ebbinghaus and Delboeuf illusions. Journal of Experimental Psychology, 95, 453-455.

Massaro, D. W., \& ANDERSon, N. H. (1971). Judgmental model of the Ebbinghaus illusion. Journal of Experimental Psychology, 89, 147-151.

O'Halloran, W. J., \& Weintraub, D. J. (1977). Delboeuf "illusions" of circle size induced via photoflash-generated afterimages. Perception \& Psychophysics, 22, 171-176.
OYAMA, T. (1960). Japanese studies on the so-called geometrical-optical illusions. Psychologia, 3, 7-20.

Pressey, A. W., \& Murray, R. (1976). Further developments in the assimilation theory of geometric illusions: The adjacency principle. Perception \& Psychophysics, 19, 536-544.

Restle, F., \& Merryman, C. (1969). Distance and an illusion of length of line. Journal of Experimental Psychology, 81, 297-302.

SEKUlER, R. (1974). Spatial vision. In M. R. Rosenzweig \& L. W. Porter (Eds.), Annual Review of Psychology (Vol. 25). Palo Alto, CA Annual Reviews.

Sjostrom, K., \& Pollack, R. H. (1972). The role of lightness contrast in determining the magnitude of the Delboeuf illusion: A rejoinder to Weintraub and Cooper. Developmental Psychology, 6, 198-200.

Weintraub, D. J. (1979). Ebbinghaus illusion: Context, contour, and age influence the judged size of a circle amidst circles. Joumal of Experimental Psychology: Human Perception \& Performance, 5, 353-364.

Weintraub, D. J. \& Cooper, L. A. (1972). Coming of age with the Delboeuf illusion: Brightness contrast, cognition, and perceptual development. Developmental Psychology, 6, 187-197.

Weintraub, D. J., Wilson, B. A., Greene, R. D., \& Palmquist, M. J. (1969). Delboeuf illusion: Displacement versus diameter, arc deletions, and brightness contrast. Journal of Experimental Psychology, 80, 505-511.

(Manuscript received November 14, 1983; revision accepted for publication June 20,1986 .) 